\title{
Influence of interferon-a treatment outcome in polycythemia vera and essential thrombocythemia by genetic polymorphism in IL28B
}

\author{
Marie Lindgren1, Helna Pettersson', Johan Westin ${ }^{3}$, Magnus Lindh ${ }^{3}$, Peter Johansson ${ }^{2,4}$, \\ Björn Andréasson ${ }^{2,4}$
}

1. Hematology Section, Medical Clinic, Kalmar, Sweden. 2. Hematology Section, Medical Clinic, NU Hospital Organization, Uddevalla, Sweden. 3. Department of Infectious Diseases, Institute of Biomedicine, Sahlgrenska Academy, University of Gothenburg, Gothenburg, Sweden. 4. Section of Hematology and Coagulation, Sahlgrenska University Hospital,

Gothenburg, Sweden

Correspondence: Björn Andréasson. Address: Hematology Section, Medical Clinic, NU Hospital Organization, Uddevalla, Sweden. Telephone: 46-104-738-100. Fax: 465-229-3232. E-mail: bjorn.andreasson@vgregion.se

Received: May 31, 2012

DOI: $10.5430 / \mathrm{jhm} . \mathrm{v} 2 \mathrm{n} 3 \mathrm{p} 18$
Accepted: June 28, $2012 \quad$ Published: September 1, 2012

URL: http://dx.doi.org/10.5430/jhm.v2n3p18

\section{Abstract}

Background: Interferon- $\alpha$ (IFN- $\alpha$ ) is a therapeutic option in high risk patients with Myeloproliferative Neoplasms (MPNs). The response to IFN- $\alpha$ treatment of chronic hepatitis $\mathrm{C}$ was shown to be strongly influenced by several related single nucleotide polymorphisms (SNP) in a region adjacent to the IL28B gene. The objective of this study was to investigate the possibility of similar influence of IL28B gene polymorphism in IFN- $\alpha$ treated MPN patients.

Methods: 20 patients with polycythemia vera (PV) or essential thrombocythemia (ET) treated with IFN- $\alpha$ included, 7 were earlier treated with hydroxyurea. Hematologic response was evaluated using the European Leukemia Net (ELN) criteria. DNA from whole blood samples was isolated using MagNA Pure LC DNA Isolation Kit I for IL28B genotyping, and the variability at rs12979860, rs12980275 and rs8099917 were determined by allelic discrimination assays using Taqman minor groove binding probes.

Results: The IFN- $\alpha$ treatment reduced platelet count $(p<0.05)$, and in patients with polycythemia vera (PV) also hemoglobin, hematocrit and white blood cell counts declined $(p<0.05)$. Hematologic response by ELN criteria was significantly influenced by the IL28B SNP. Among 20 patients with PV or ET, the CC genotype at rs12979860 was found in 8/11 patients (73\%) with hematologic CR as compared with 1/9 (11\%) with PR or NR $(p=0.010)$.

Conclusion: The results in this pilot study indicate that variation in IL28B genotype might influence hematologic response in IFN- $\alpha$ treated MPN patients.

\section{Key words}

Polycythemia vera, Essential thrombocythemia, Interferon, IL28B, Hematologic response 


\section{Introduction}

The Philadelphia-chromosome negative myeloproliferative neoplasms (MPNs) are a heterogeneous family of clonal stem cell disorders with overlapping phenotypic and pathological features, the main or classic entities being polycythemia vera (PV), essential thrombocythemia (ET) and primary myelofibrosis (PMF). The identification of JAK2 V617F mutation is central in the diagnostics of Philadelphia-chromosome negative MPNs, and is found positive in approximately $95 \%$ of patients with PV and in $60 \%$ of patients with ET and PMF ${ }^{[1]}$.

Interferon- $\alpha(\operatorname{IFN}-\alpha)$ is a therapeutic option in MPNs when cytoreduction is indicated. INF- $\alpha$ suppresses growth of multipotent hematopoietic progenitor cells and has the potential of normalizing myeloproliferation, reducing vascular events, and lowering the JAK2 V617F allele burden ${ }^{[2]}$. Recent reports have also implicated a possibility of reversing bone marrow histopathologic abnormalities in PV and $\mathrm{PMF}^{[3]}$. In the Nordic MPN Group guidelines IFN- $\alpha$ is the recommended first line therapy for younger patients with PV and ET, with high risk criteria, based on its lack of leukemogenesis together with the potential of hematologic and molecular response ${ }^{[4]}$. IFN- $\alpha$ treatment may be limited due to its lack of efficacy and patients concerns about tolerability due to adverse side effects (e.g. flu-like symptoms and neuropsychiatric side effects) and the need for subcutaneous administration ${ }^{[3,5]}$. The availability of pegylated (peg) forms has increased the feasibility of IFN- $\alpha$ treatment due to less toxicity and a more favorable pharmacokinetic profile.

IFN- $\alpha$, together with IFN- $\beta$, belongs to the family of type I IFNs, is an approved drug for treatment of various cancers including hairy cell leukemia, chronic myeloic leukemia, multiple myeloma, follicular lymphoma, carcinoid syndrome and malignant melanoma ${ }^{[6]}$. Its use as an anti-tumor treatment remains limited in contrast to its widespread use as an antiviral agent. In chronic hepatitis C virus (HCV) infection, the standard of care treatment includes pegIFN- $\alpha$ in combination with ribavirin, with a sustained viral response (SVR) occurring in approximately $50 \%$ of the patients ${ }^{[7,8]}$. HCV genotype is well established as a strong predictor for treatment response, genotype 1 showing the lowest rates of SVR, and is a primary determinant of treatment duration ${ }^{[9,10]}$.

Four genome-wide association studies (GWAS) have demonstrated several linked single nucleotide polymorphisms (SNPs) in the close proximity of the region of interleukin-28B (IL28B) as being highly predictive of the response to pegIFN- $\alpha$ and ribavirin treatment for HCV infection ${ }^{[11]}$. The first and largest of these studies, reported by Ge et al in 2009, identified the C allele at rs12979860 as a strong predictor of SVR in HCV genotype 1-infected patients ${ }^{[10]}$. Patients homozygous for the $\mathrm{C}$ allele, i.e. carrying CC genotype, had a 2-fold higher SVR rate as compared with those with CT or TT genotype. The frequency of CC genotype is more common in European than in African populations, which partly explains the difference in HCV treatment response between these two groups. The CC genotype at rs12979860 was also favorable in terms of a 3-fold greater likelihood of spontaneous clearance of HCV infection compared to patients carrying the TC or TT genotype ${ }^{[10,12]}$. Subsequent GWAS showed that treatment response was significantly influenced by other IL28B SNPs, in particular rs8099917 ${ }^{[13-15]}$ and rs12980275 ${ }^{[13]}$. Thus, patients with genotype TT at rs8099917 and genotype AA at rs12980275 have been shown to have a higher SVR rate in pegIFN- $\alpha$ and ribavirin treated patients with HCV infection. Considerable co-variation has been shown between rs12979860 and rs8099917 ${ }^{[15]}$, as well as between rs12979860 and rs12980275 ${ }^{[16]}$.

There are to this date no reports regarding SNP variations in the IL28B region and IFN- $\alpha$ treatment outcome in myeloid malignancies. The IFN- $\alpha$ treatment time has not limitations, but side effects may lead to discontinuation of treatment ${ }^{[17]}$. A valid biomarker to support therapy decision regarding the choice of cytoreductive treatment would be of great value. With the knowledge of the genetic biomarkers in IL28B and IFN- $\alpha$ treatment outcome in HCV genotype 1, we have performed a pilot study on IFN- $\alpha$ in 20 patients with PV or ET.

The aim of the present study was to compare the hematologic response with respect to the IL28B polymorphism at rs12979860, rs8099917 and rs12980275. 


\section{Material and methods}

\subsection{Patients and study design}

This study includes 12 patients with ET and 8 with PV, currently or previously treated with IFN- $\alpha$, followed up at Sahlgrenska University Hospital and Uddevalla Hospital. The gender, ages, and results of routine blood tests are described in Table 1. All the patients satisfy the 2008 WHO diagnostic criteria for ET or PV, including those who were diagnosed before $2008^{[18]}$. IFN- $\alpha$ was chosen as the best treatment for these high risk patients, i.e. age over 60 years, thrombotic complications and/or platelets $>1500 \times 10^{9} / \mathrm{L}$, with the intention of long term treatment in the absence of side effects and complications. 10 patients received IFN- $\alpha 2 b$ and 10 patients received IFN- $\alpha 2 a$, the majority of them had or have had subcutaneous injections with 3M units 3 times a week and $135 \mu$ g weekly for IFN- $\alpha 2 b$ and IFN- $\alpha 2 a$, respectively. Seven patients had prior to IFN- $\alpha$ therapy been treated with hydroxyurea and the majority of PV patients had been phlebotomized. The length of treatment in months varies greatly, the median time on IFN- $\alpha$ therapy was 50 months (range 6-240). For the patients who reached European Leukemia Net (ELN) criteria CR the median treatment time was 32 months (range 5-138), whereas the patients who did not reach CR had been on IFN- $\alpha$ for a median time of 60 months (range 9-240).

Table 1. Demographic data and peripheral blood counts for 20 patients with PV and ET treated with interferon (median and ranges are given)

\begin{tabular}{lllll}
\hline & PV $\mathbf{n = 8}$ & & ET $\mathbf{n}=\mathbf{1 2}$ & \\
\hline Gender (male/female) & $3 / 5$ & & $5 / 7$ & \\
Age at diagnosis & 48 & & 52 & \\
& $(29-58)$ & & $(31-62)$ & \\
JAK2 V617F/wt & $6 / 0$ & & $4 / 5$ & \\
HU treatment prior to IFN & 4 & After IFN & Before IFN & 127 \\
& Before IFN & $126^{*}$ & 144 & $(100-157)$ \\
Hemoglobin (g/L) & 145 & $(104-147)$ & $(101-161)$ & 38.5 \\
& $(127-171)$ & $42^{*}$ & 45 & $(36-46)$ \\
Hematocrit $(\%)$ & 47 & $(32-46)$ & $(30-49)$ & 5.0 \\
& $(44-54)$ & $4.7^{*}$ & 8.4 & $(2.3-8.1)$ \\
White blood cells $\left(\times 10^{9} / \mathrm{L}\right)$ & 8.4 & $(2.9-8.8)$ & $(3.2-11.4)$ & $364^{*}$ \\
& $(5.8-14.6)$ & $371^{*}$ & 1070 & $(187-654)$ \\
\hline Platelets $\left(\times 10^{9} / \mathrm{L}\right)$ & 936 & $(208-535)$ & $(291-1580)$ & \\
\hline
\end{tabular}

$*=p<0.05($ Log Rank test $)$, difference between before and after interferon treatment

Abbreviations: $\mathrm{PV}=$ polycythemia vera, $\mathrm{ET}=$ essential thrombocythemia, wt= wild type, $\mathrm{HU}=$ hydroxyurea, $\mathrm{IFN}=$ interferon

Medical charts of these patients were reviewed, treatment duration of IFN- $\alpha$ was noted and laboratory data corresponding to the period of time they were undergoing interferon treatment were evaluated. The ELN criteria were used to evaluate response to treatment of PV and $\mathrm{ET}^{[19]}$. Laboratory data and time to best clinical sustained response were recorded.

A large proportion of the patients were diagnosed, and IFN- $\alpha$ therapy was initiated before the discovery of JAK2 mutation in 2005, making meaningful evaluation of the molecular response to treatment not possible in this study as no pre-treatment allele burden was available in this group.

\subsection{Genetic analyses}

A $5 \mathrm{~mL}$ peripheral blood sample was obtained from each of the included patients. DNA from whole blood samples was isolated using MagNA Pure LC DNA Isolation Kit I (Roche diagnostics, Mannheim, Germany) for IL28B genotyping. The variability at rs12979860, rs12980275 and rs8099917 were determined by allelic discrimination assays using Taqman minor groove binding probes, using primers and probes described in Table 2. Briefly, the analysis was performed by first 
running a two-step PCR (15 s at 95 _C; 60 s at 60 _C) on an ABI 7300 instrument (Applied Biosystems, Foster City, CA, USA), followed by a post-PCR read of fluorescence intensity from the two fluorophores to allow allelic discrimination.

Table 2. Primers and probes used for the determination of IL28B genotype

\begin{tabular}{lll}
\hline snp & & \\
\hline rs12979860 & Forward primer & GTGCCTGTCGTGTACTGAACCA \\
& Fam probe & FAM-CCTGGTTCGCGCCTT-MGB \\
& Vic probe & VIC-CCTGGTTCACGCCT-MGB \\
& Reverse primer & AGCGCGGAGTGCAATTCA \\
rs8099917 & Forward primer & TGTTCCTCCTTTTGTTTTCCTTTC \\
& Fam probe & FAM-TGTGAGCAATGTCACCCA-MGB \\
& Vic probe & VIC-TGTGAGCAATTTCACCCAA-MGB \\
rs12980275 & Reverse primer & CAAACTGTATACAGCATGGTTCCAA \\
& Forward primer & CTACATGAGGTGCTGAGAGAAGTCA \\
& Fam probe & FAM-ATTCCTAGAAACAGACGTGTC-MGB \\
& Vic probe & VIC-CCTAGAAACGGACGTGTC-MGB \\
& Reverse primer & TCCTATTAACCCCTCCCGCTAC \\
\hline
\end{tabular}

\subsection{Ethical considerations}

This study was approved by the Ethical Committee of the "Västra Götaland Regionen”. All participants gave their written informed consent.

\subsection{Statistical methods}

Routine statistical methods for mean, median and range were used. Log Rank test was used when two groups were compared. Fisher's exact test was used to compare response rates.

\section{Results}

The peripheral blood values before IFN treatment was initiated showed no significant differences between the 12 ET patients and the $8 \mathrm{PV}$ patients, data are shown in Table 1. In the ET patients, the platelet concentration, after IFN treatment, was significantly reduced from a mean of 1070 to $364(p=0.002)$. The PV patients had a significant reduction of mean hemoglobin concentration, hematocrit, white blood cell concentration and platelet concentration after IFN treatment ( $p=0.021,0.009,0.021$ and 0.028 , respectively).

Three different SNPs were analyzed. The rs12979860 analysis showed that 9 patients had the CC genotype, 10 patients had the CT and one patient had TT genotype. As to rs8099917, 12 patients had the TT and 8 had the TG genotype. Corresponding genotypes for rs12980275 were 10 patients with AA, 9 with AG and one patient with GG. Nine patients had the CTA/CTA haplotype while 11 patients had other combinations.

Among the 20 MPN patients 5 with PV and 6 with ET reached hematologic CR with criteria stipulated by ELN. Two patients with PV and 6 with ET had PR and one PV patient was NR. For the 11 patients who reached CR the mean platelet concentration was significantly reduced from 1019 to $326(p<0.001)$, whereas the corresponding means for the patients not reaching CR were 785 and 470 ( $p=0.228$ ). The mean platelet concentrations prior to IFN treatment in the group of patients reaching $\mathrm{CR}$ did not differ significantly from the PR/NR group $(p=0.263)$. On the other hand the mean platelet concentration after treatment differed significantly after IFN treatment $(p=0.029)$. 
Ten of the 20 MPN patients stopped IFN treatment due to side effects, 5 of these had reached CR criteria and 5 were in PR. Out of the 10 patient who stopped treatment, 5 had been treated with IFN- $\alpha 2 \mathrm{a}$ and 5 had been on IFN- $\alpha 2 \mathrm{~b}$ during the main treatment time, two patients tried both types of IFN. The main adverse symptoms were fatigue (5), depression (2), myalgia/flu like symptoms (2), neutropenia (1), alopecia (1) and anemia (1). There were no significant differences with regard to IL28B genotype between patients who had stopped treatment and the ones who were still on IFN.

Variations in the investigated SNPs according to treatment outcome are displayed in Table 3. Out of the 9 PV and ET patients with CC genotype in rs12979860 8 obtained CR after IFN therapy, whereas one patient was in PR. Among the patients with genotype CT or TT only three had CR, 7 had PR and one had NR. The difference between the CC vs. the non-CC group was statistically significant $(p=0.010)$. The combined favorable haplotype of the three investigated SNPs (CTA/CTA) showed identical CR rates as the CC genotype $(p=0.010) .10 \mathrm{PV}$ and ET patients had genotype AA in rs12980275, 8 of them reached CR and 2 PR. Only 3 out of the 10 patients with AG or GG genotype were in CR after IFN- $\alpha$ treatment. This difference did not quite reach statistical significance (0.070). As to rs8099917, corresponding difference between the TT and non-TT groups did not reach statistical significance ( $p=0.175)$.

Eleven of the PV and ET patients had been exposed to hydroxyurea (HU) therapy prior to or after IFN- $\alpha$ treatment. Two patients were intolerant to HU (one with CC genotype and one with CT); one patient with CT genotype needed a combination of HU and IFN to reach PR. Three patients reached a better hematologic response on HU compared to IFN- $\alpha$, 3 had unchanged response and 2 had better response to IFN- $\alpha$. Three of the 5 patients who reached CR on HU therapy had CC genotype and 2 had CT.

Table 3. Hematologic response (ELN criteria) during interferon treatment in 20 patients with polycythemia vera and essential thrombocythemia with regard to IL28B polymorphism

\begin{tabular}{|c|c|c|c|c|c|c|c|}
\hline & \multicolumn{2}{|c|}{ PV $n=8$} & \multicolumn{2}{|c|}{ ET $n=12$} & \multicolumn{3}{|c|}{ All $\mathbf{n}=\mathbf{2 0}$} \\
\hline IL28B genotype & $\mathrm{CR}$ & PR or NR & CR & PR or NR & $\mathrm{CR}$ & PR or NR & $p$ \\
\hline \multicolumn{8}{|l|}{ rs 12979860} \\
\hline CC & 4 & 0 & 4 & 1 & 8 & 1 & \multirow{2}{*}{0.010} \\
\hline Non-CC & 1 & 3 & 2 & 5 & 3 & 8 & \\
\hline \multicolumn{8}{|l|}{ rs 8099917} \\
\hline $\mathrm{TT}$ & 4 & 0 & 4 & 3 & 8 & 3 & \multirow{2}{*}{0.175} \\
\hline Non-TT & 1 & 3 & 2 & 3 & 3 & 6 & \\
\hline \multicolumn{8}{|l|}{ rs 12980275} \\
\hline AA & 3 & 1 & 4 & 2 & 8 & 2 & \multirow{2}{*}{0.070} \\
\hline Non-AA & 2 & 2 & 2 & 4 & 3 & 7 & \\
\hline \multicolumn{8}{|l|}{ Haplotype } \\
\hline CTA/CTA & 4 & 0 & 4 & 1 & 8 & 1 & \multirow{2}{*}{0.010} \\
\hline Non-CTA/CTA & 1 & 3 & 2 & 5 & 3 & 8 & \\
\hline
\end{tabular}

Abbreviations: $\mathrm{PV}=$ polycythemia vera, $\mathrm{ET}=$ essential thrombocythemia, $\mathrm{CR}=$ complete response (ELN criteria), $\mathrm{PR}=$ partial response ( $\mathrm{ELN}$ criteria), $\mathrm{NR}=\mathrm{no}$ response (ELN criteria), $p=$ significance level all patients are compared ( $\mathrm{n}=20$ ) (Fisher's exact test).

\section{Discussion}

Since the discovery of polymorphism in close proximity of the region of IL28B in 2009 the analysis of the genotype at rs12979860 has rapidly become available and used in routine clinical practice as a base parameter together with viral load and liver fibrosis stage as a significant pre-treatment predictor of response to therapy in HCV genotype $1^{[20]}$.

In this study 20 MPN patients who underwent treatment with recombinant IFN therapy were evaluated with respect to hematologic response, according to the ELN criteria, and IL28B gene polymorphism. Nine out of the 23 patients (39\%) had the CC genotype at rs12979860, which in HCV treatment is favorable. The corresponding frequencies for TT 
genotype at rs 8099917 and AA genotype at rs12980275 were $61 \%$ and 43\%, respectively. The studied SNPs are part of the normal human genome and the genotype frequencies are found to vary in different populations, the frequencies in our cohort correspond well with what is reported for a white population ${ }^{[21]}$.

Among the $20 \mathrm{ET}$ and PV patients treated with IFN the hematologic CR rate was $89 \%$ in the patient group with genotype CC at rs12979860 compared to $27 \%$ with the CT or TT genotype. This difference was statistically significant $(p=0.010)$. The corresponding CR rate for patients with AA genotype at rs12980275 was 80\% compared to $30 \%$ for patients with AG or GG genotype, this difference did not quite reach statistical significance $(p=0.070)$. Further, the patients with TT genotype at rs8099917 reached CR in 73\% compared to 33\% in TG genotype group ( $p=0.175)$. When combining the three evaluated SNPs, the haploid CTA/CTA showed the same CR rates as the genotype CC at rs12979860 ( $p=0.010)$. (Table 3) The number of patients with available JAK2 mutation status was too small to make any conclusions, several patients were diagnosed and started treatment before the identification of JAK2 V617F mutation in 2005.

During IFN treatment the mean platelet concentrations from both ET and PV patients decreased significantly (Table 1). There were no significant differences in peripheral blood counts before IFN therapy was initiated when the group of patients with genotype CC at rs12979860 was compared to the ones with CT or TT genotype. Also, the decrease of platelet concentration was significant for both groups during IFN treatment. Although the number of patients is limited, the effect on CR rate seems to be linked to the IFN therapy. Thus, 10 of the patients had also been treated with HU, out of these 8 patients had HU therapy for long enough time to evaluate hematologic response; 3 patients got a better response on HU, 2 experienced a poorer response grade, while 3 had an unchanged response grade.

Two types of recombinant IFN were used in the treatment; INF- $\alpha 2 a$ and INF- $\alpha 2 b$. There was no significant difference with respect to hematologic response rate between the 2 types. Neither did we see any differences in side-effects among the 10 patients where IFN therapy was stopped, 5 had been treated with IFN- $\alpha 2$ a and 5 had been on IFN- $\alpha 2 b$. The main causes for ending therapy were fatigue, depression and myalgia/flu like symptoms, which are frequently reported symptoms.

IL28B, discovered and first described in $2003^{[22,23]}$, belongs to the family of interferon- $\lambda$ (IFN- $\lambda$ ) and classified as type III IFN. These cytokines are distantly related to the type I IFNs ( $\alpha$ and $\beta$ ) and the IL-10 family. Although type I IFNs and type III IFNs use different receptor complexes the signaling through either receptor complex results in the same JAK-STAT signal transduction cascade ${ }^{[24,25]}$. Thus, in spite of the fact that the type I and type III IFNs influence two independent cell surface receptors they induce the same gene subset and exhibit similar biological activities such as inhibition of viral replication, cellular growth inhibition, and apoptosis ${ }^{[26]}$. In contrast to IFN- $\alpha$ receptors, which are expressed on most cell types, IFN- $\lambda$ receptors are expressed mainly on epithelial cells and specific subsets of immune cells ${ }^{[25]}$. It might be that synergistic effects between the treatment type I IFN and the favorable type III IFN genotype are responsible for the response benefits found in this study ${ }^{[27]}$.

We conclude that, even if the number of patients in this pilot study is small, IL28B gene polymorphism could be a valuable predictor for IFN treatment response in MPN patients. Larger studies have to be performed to confirm these findings.

\section{Acknowledgements}

The study was supported by grants from "FOU NU-hospital organization”.

\section{Authorship contributions}

ML-n and BA designed research. JW and ML-h contributed vital analytical tools. HP, PJ and BA collected data. BA analyzed data. ML-n, HP, JW, ML-h, PJ and BA wrote the manuscript. None of the authors have any relevant disclosures. 


\section{References}

[1] Campbell PJ, Green AR. The myeloproliferative disorders. The New England journal of medicine. 2006; 355: $2452-66$. PMid:17151367 http://dx.doi.org/10.1056/NEJMra063728

[2] Kiladjian JJ, Cassinat B, Chevret S, Turlure P, Cambier N, Roussel M, et al. Pegylated interferon-alfa-2a induces complete hematologic and molecular responses with low toxicity in polycythemia vera. Blood. 2008; 112: 3065-72. PMid:18650451 http://dx.doi.org/10.1182/blood-2008-03-143537

[3] Kiladjian JJ, Mesa RA, Hoffman R. The renaissance of interferon therapy for the treatment of myeloid malignancies. Blood. 2011; 117: 4706-15. PMid:21389325 http://dx.doi.org/10.1182/blood-2010-08-258772

[4] MPNstudygroup N. Nationella riktlinjer [Internet]. 2009. Available from: http://www.sfhem.se/Filarkiv/Nationella-riktlinjer/(offset)/10

[5] Hasselbalch HC. A new era for IFN-alpha in the treatment of Philadelphia-negative chronic myeloproliferative neoplasms. Expert review of hematology. 2011; 4: 637-55.

[6] Bracarda S, Eggermont AM, Samuelsson J. Redefining the role of interferon in the treatment of malignant diseases. Eur J Cancer 2010; 46: 284-97. PMid:19906524 http://dx.doi.org/10.1016/j.ejca.2009.10.013

[7] Lindh M, Lagging M, Arnholm B, Eilard A, Nilsson S, Norkrans G, et al. IL28B polymorphisms determine early viral kinetics and treatment outcome in patients receiving peginterferon/ribavirin for chronic hepatitis C genotype 1. Journal of viral hepatitis. 2011; 18: e325-31. PMid:21692944 http://dx.doi.org/10.1111/j.1365-2893.2010.01425.x

[8] Kurosaki M, Tanaka Y, Nishida N, Sakamoto N, Enomoto N, Honda M, et al. Pre-treatment prediction of response to pegylated-interferon plus ribavirin for chronic hepatitis C using genetic polymorphism in IL28B and viral factors. Journal of hepatology. 2011; 54: 439-48. PMid:21129805 http://dx.doi.org/10.1016/j.jhep.2010.07.037

[9] Hayashi K, Katano Y, Honda T, Ishigami M, Itoh A, Hirooka Y, et al. Association of interleukin 28B and mutations in the core and NS5A region of hepatitis C virus with response to peg-interferon and ribavirin therapy. Liver Int. 2011; 31: 1359-65. PMid:21745312 http://dx.doi.org/10.1111/j.1478-3231.2011.02571.x

[10] Ge D, Fellay J, Thompson AJ, Simon JS, Shianna KV, Urban TJ, et al. Genetic variation in IL28B predicts hepatitis C treatment-induced viral clearance. Nature. 2009; 461: 399-401. PMid:19684573 http://dx.doi.org/10.1038/nature08309

[11] Dill MT, Duong FH, Vogt JE, Bibert S, Bochud PY, Terracciano L, et al. Interferon-induced gene expression is a stronger predictor of treatment response than IL28B genotype in patients with hepatitis C. Gastroenterology. 2011; 140: 1021-31. PMid:21111740 http://dx.doi.org/10.1053/j.gastro.2010.11.039

[12] Thomas DL, Thio CL, Martin MP, Qi Y, Ge D, O'Huigin C, et al. Genetic variation in IL28B and spontaneous clearance of hepatitis C virus. Nature. 2009; 461: 798-801. PMid:19759533 http://dx.doi.org/10.1038/nature08463

[13] Tanaka Y, Nishida N, Sugiyama M, Kurosaki M, Matsuura K, Sakamoto N, et al. Genome-wide association of IL28B with response to pegylated interferon-alpha and ribavirin therapy for chronic hepatitis C. Nature genetics. 2009; 41: 1105-9. PMid:19749757 http://dx.doi.org/10.1038/ng.449

[14] Suppiah V, Moldovan M, Ahlenstiel G, Berg T, Weltman M, Abate ML, et al. IL28B is associated with response to chronic hepatitis C interferon-alpha and ribavirin therapy. Nature genetics. 2009; 41: 1100-4. PMid:19749758 http://dx.doi.org/10.1038/ng.447

[15] Rauch A, Kutalik Z, Descombes P, Cai T, Di Iulio J, Mueller T, et al. Genetic variation in IL28B is associated with chronic hepatitis C and treatment failure: a genome-wide association study. Gastroenterology. 2010; 138: 1338-45, 45 e1-7.

[16] Bochud PY, Bibert S, Negro F, Haagmans B, Soulier A, Ferrari C, et al. IL28B polymorphisms predict reduction of HCV RNA from the first day of therapy in chronic hepatitis C. Journal of hepatology. 2011; 55: 980-8. PMid:21354446 http://dx.doi.org/10.1016/j.jhep.2011.01.050

[17] Samuelsson J, Hasselbalch H, Bruserud O, Temerinac S, Brandberg Y, Merup M, et al. A phase II trial of pegylated interferon alpha-2b therapy for polycythemia vera and essential thrombocythemia: feasibility, clinical and biologic effects, and impact on quality of life. Cancer. 2006; 106: 2397-405. PMid:16639737 http://dx.doi.org/10.1002/cncr.21900

[18] Tefferi A, Vardiman JW. Classification and diagnosis of myeloproliferative neoplasms: the 2008 World Health Organization criteria and point-of-care diagnostic algorithms. Leukemia. 2008; 22: 14-22. PMid:17882280 http://dx.doi.org/10.1038/sj.leu.2404955

[19] Barosi G, Birgegard G, Finazzi G, Griesshammer M, Harrison C, Hasselbalch HC, et al. Response criteria for essential thrombocythemia and polycythemia vera: result of a European LeukemiaNet consensus conference. Blood. 2009; 113: 4829-33. PMid:19278953 http://dx.doi.org/10.1182/blood-2008-09-176818 
[20] Ghany MG, Nelson DR, Strader DB, Thomas DL, Seeff LB. An update on treatment of genotype 1 chronic hepatitis C virus infection: 2011 practice guideline by the American Association for the Study of Liver Diseases. Hepatology. 2011; 54: 1433-44. PMid:21898493 http://dx.doi.org/10.1002/hep.24641

[21] Melis R, Fauron C, McMillin G, Lyon E, Shirts B, Hubley LM, et al. Simultaneous genotyping of rs12979860 and rs8099917 variants near the IL28B locus associated with HCV clearance and treatment response. J Mol Diagn. 2011; $13: 446-51$. PMid:21704279 http://dx.doi.org/10.1016/j.jmoldx.2011.03.008

[22] Kotenko SV, Gallagher G, Baurin VV, Lewis-Antes A, Shen M, Shah NK, et al. IFN-lambdas mediate antiviral protection through a distinct class II cytokine receptor complex. Nature immunology. 2003; 4: 69-77. PMid:12483210 http://dx.doi.org/10.1038/ni875

[23] Sheppard P, Kindsvogel W, Xu W, Henderson K, Schlutsmeyer S, Whitmore TE, et al. IL-28, IL-29 and their class II cytokine receptor IL-28R. Nature immunology. 2003; 4: 63-8. PMid:12469119 http://dx.doi.org/10.1038/ni873

[24] Donnelly RP, Kotenko SV. Interferon-lambda: a new addition to an old family. J Interferon Cytokine Res. 2010 ; 30: 555-64.

[25] Kotenko SV. IFN-lambdas. Current opinion in immunology. 2011; 23: 583-90. PMid:21840693 http://dx.doi.org/10.1089/jir.2010.0078

[26] Li M, Liu X, Zhou Y, Su SB. Interferon-lambdas: the modulators of antivirus, antitumor, and immune responses. Journal of leukocyte biology. 2009; 86: 23-32. PMid:19304895 http://dx.doi.org/10.1189/jlb.1208761

[27] Maher SG, Sheikh F, Scarzello AJ, Romero-Weaver AL, Baker DP, Donnelly RP, et al. IFNalpha and IFNlambda differ in their antiproliferative effects and duration of JAK/STAT signaling activity. Cancer biology \& therapy. 2008; 7: 1109-15. http://dx.doi.org/10.4161/cbt.7.7.6192 